\title{
The crystal structure of bis(ferrocenecarboxylato- $\mathrm{K}^{2} \mathrm{O}, \mathrm{O}^{\prime}$ ) bis[4-(dimethylamino)pyridine- $\mathrm{KN}$ ] copper(II) - acetonitrile(1/2), $\mathrm{C}_{40} \mathrm{H}_{44} \mathrm{CuO}_{4} \mathrm{Fe}_{2} \mathrm{~N}_{6}$
}

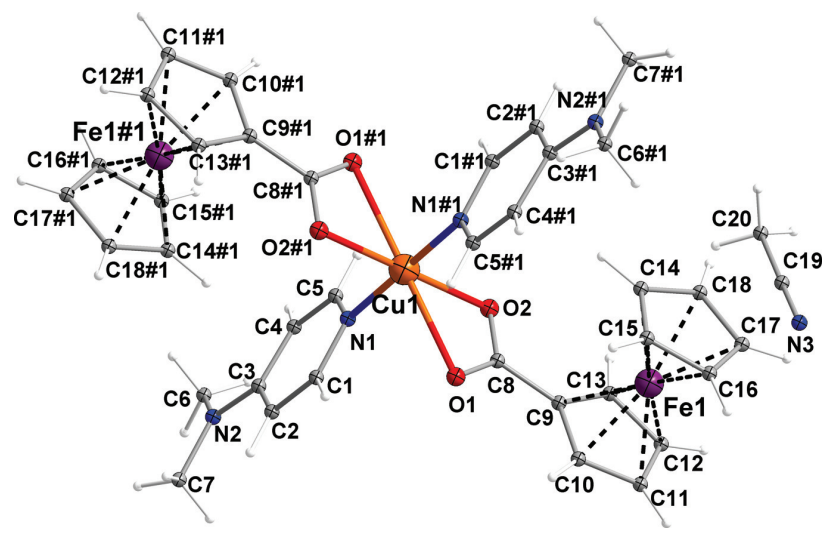

https://doi.org/10.1515/ncrs-2019-0038

Received January 14, 2019; accepted March 9, 2019; available online April 2, 2019

\begin{abstract}
$\mathrm{C}_{40} \mathrm{H}_{44} \mathrm{CuO}_{4} \mathrm{Fe}_{2} \mathrm{~N}_{6}$, triclinic, $P \overline{1}$ (no. 2), $a=7.4758(4) \AA$, $b=7.7447(5) \AA, \quad c=16.2929(10) \AA, \quad \alpha=87.111(2)^{\circ}$, $\beta=85.196(2)^{\circ}, \quad \gamma=89.736(2)^{\circ}, \quad V=938.82(10) \AA^{3}, \quad Z=1$, $R_{\mathrm{gt}}(F)=0.0672, w R_{\text {ref }}\left(F^{2}\right)=0.1316, T=150(2) \mathrm{K}$.
\end{abstract}

CCDC no.: 1902121

Table 1 contains crystallographic data and Table 2 contains the list of the atoms including atomic coordinates and displacement parameters.

\section{Source of material}

Ferrocenecarboxylic acid (0.069 g, $0.3 \mathrm{mmol})$ was dissolved in a methanol solution $(6 \mathrm{~mL})$ of $\mathrm{Cu}\left(\mathrm{CH}_{3} \mathrm{CN}\right)_{4} \mathrm{BF}_{4}(0.095 \mathrm{~g}$,

\footnotetext{
*Corresponding author: Xiao-Wei Yan, College of Materials and Environmental Engineering, and Guangxi Key Laboratory of Calcium Carbonate Resources Comprehensive Utilization, Hezhou University, No. 18 West Ring Road, Hezhou, Guangxi 542800, P.R. China, e-mail: yanxiaoweiw@163.com

Su-Juan Wang and Dong-Jian Zhu: College of Food and Bioengineering, Hezhou University, No. 18 West Ring Road, Hezhou, Guangxi 542899, P.R. China

Ai-Shan Ren: College of Materials and Environmental Engineering, and Guangxi Key Laboratory of Calcium Carbonate Resources Comprehensive Utilization, Hezhou University, No. 18 West Ring Road, Hezhou, Guangxi 542800, P.R. China
}

Table 1: Data collection and handling.

\begin{tabular}{ll}
\hline Crystal: & Blue block \\
Size: & $0.25 \times 0.13 \times 0.12 \mathrm{~mm}$ \\
Wavelength: & Mo $K \alpha$ radiation $(0.71073 \AA)$ \\
$\mu:$ & $1.37 \mathrm{~mm}^{-1}$ \\
Diffractometer, scan mode: & Bruker APEX-III CMOS, $\varphi$ and $\omega$ \\
$\theta_{\max }$, completeness: & $26.2^{\circ}, 99 \%$ \\
$N\left(h k l_{\text {measured }}, N\left(h k l l_{\text {unique }}, R_{\text {int }}:\right.\right.$ & $3819,3819,0.064$ \\
Criterion for $I_{\text {obs }}, N(h k l)_{\mathrm{gt}}:$ & $I_{\text {obs }}>2 \sigma\left(I_{\text {obs }}\right), 3089$ \\
$N(\text { param })_{\text {refined }}:$ & 246 \\
Programs: & SHELX [1], Diamond [2], Bruker [3] \\
\hline
\end{tabular}

$0.3 \mathrm{mmol}$ ) under ultrasonication. To the resulting yellow solution triethylamine $(42 \mu L, 0.3 \mathrm{mmol})$ was added, after $10 \mathrm{~min}$ 4-(dimethylamino)pyridine (DMAP) $(0.122 \mathrm{~g}$, $0.6 \mathrm{mmol}$ ) was added under ultrasonication. The cloudy mixture was sealed and heated to $70{ }^{\circ} \mathrm{C}$ for $20 \mathrm{~h}$. After cooled to room temperature, it was filtered, and the filtrate was evaporated at $5^{\circ} \mathrm{C}$. After 2 weeks, blue block crystals of title complex formed.

\section{Experimental details}

All hydrogen atoms were positioned geometrically and allowed to ride on their parent atoms.

\section{Comment}

Heterometallic complexes have been widely applied for medical use [4, 5]. Although ferrocene and its derivatives are popular molecules for biological applications [6, 7], there are few examples of heterometallic complexes to be used in medical useage. Preparation and revelation of heterometallic complexes constructed by reaction of ferrocene derivatives with other metals $[8,9]$ are the key problems for their application in medical use.

Here, we mix ferrocenecarboxylic acid ( $\mathrm{FcCOOH})$ with $\mathrm{Cu}\left(\mathrm{CH}_{3} \mathrm{CN}\right) \mathrm{BF}_{4}$ under the assistance of 4-(dimethylamino) pyridine (DMAP) to get the title complex. The asymmetric unit contains 0.5 copper ions, one ferrocenecarboxylato(1), one DMAP and one free $\mathrm{CH}_{3} \mathrm{CN}$. In the mononuclear title complex, the $\mathrm{Cu}^{\mathrm{II}}$ ion, located on a crystallographic inversion centre, is six coordinated by two $\mathrm{N}$ atoms of two DMAP ligands in apical positions and four $\mathrm{O}$ atoms from 
Table 2: Fractional atomic coordinates and isotropic or equivalent isotropic displacement parameters $\left(\AA^{2}\right)$.

\begin{tabular}{|c|c|c|c|c|}
\hline Atom & $x$ & $y$ & $z$ & $U_{\text {iso }}{ }^{*} / U_{\text {eq }}$ \\
\hline Cu1 & 0.500000 & 0.500000 & 0.500000 & $0.0106(3)$ \\
\hline Fe1 & $0.49974(11)$ & $0.53615(11)$ & $0.17113(5)$ & $0.0148(3)$ \\
\hline 01 & $0.4377(6)$ & $0.7117(5)$ & $0.3866(3)$ & $0.0176(9)$ \\
\hline 02 & $0.6355(5)$ & $0.4967(5)$ & $0.3874(2)$ & $0.0136(9)$ \\
\hline N1 & $0.6848(7)$ & $0.6512(6)$ & $0.5384(3)$ & $0.0134(11)$ \\
\hline $\mathrm{N} 2$ & $1.0485(7)$ & $0.9742(6)$ & $0.6395(3)$ & $0.0171(11)$ \\
\hline N3 & $0.8868(14)$ & $0.2079(13)$ & $0.0418(6)$ & $0.079(3)$ \\
\hline C1 & $0.6579(8)$ & $0.8233(7)$ & $0.5423(4)$ & $0.0145(13)$ \\
\hline $\mathrm{H} 1$ & 0.552145 & 0.870335 & 0.521536 & $0.017^{\star}$ \\
\hline $\mathrm{C} 2$ & $0.7736(8)$ & $0.9354(8)$ & $0.5745(4)$ & $0.0163(13)$ \\
\hline $\mathrm{H} 2$ & 0.745742 & 1.055002 & 0.576625 & $0.020^{*}$ \\
\hline C3 & $0.9345(8)$ & $0.8695(7)$ & $0.6042(3)$ & $0.0133(12)$ \\
\hline C4 & $0.9681(8)$ & $0.6920(8)$ & $0.5965(4)$ & $0.0160(13)$ \\
\hline $\mathrm{H} 4$ & 1.077012 & 0.642011 & 0.612456 & $0.019^{\star}$ \\
\hline C5 & $0.8405(8)$ & $0.5922(7)$ & $0.5656(4)$ & $0.0138(13)$ \\
\hline H5 & 0.863843 & 0.471787 & 0.563092 & $0.017^{\star}$ \\
\hline C6 & $1.2196(9)$ & $0.9130(9)$ & $0.6630(5)$ & $0.0282(16)$ \\
\hline $\mathrm{H} 6 \mathrm{~A}$ & 1.300468 & 1.011460 & 0.665579 & $0.042^{\star}$ \\
\hline $\mathrm{H} 6 \mathrm{~B}$ & 1.271192 & 0.834061 & 0.622221 & $0.042^{\star}$ \\
\hline $\mathrm{H} 6 \mathrm{C}$ & 1.203682 & 0.851880 & 0.717251 & $0.042^{\star}$ \\
\hline $\mathrm{C} 7$ & $1.0124(8)$ & $1.1594(8)$ & $0.6438(4)$ & $0.0208(14)$ \\
\hline $\mathrm{H} 7 \mathrm{~A}$ & 1.080849 & 1.206704 & 0.686237 & $0.031 *$ \\
\hline H7B & 0.883876 & 1.177598 & 0.657833 & $0.031^{\star}$ \\
\hline $\mathrm{H} 7 \mathrm{C}$ & 1.048235 & 1.217958 & 0.590284 & $0.031 *$ \\
\hline C8 & $0.5558(7)$ & $0.6218(7)$ & $0.3505(3)$ & $0.0116(12)$ \\
\hline C9 & $0.6085(8)$ & $0.6575(8)$ & $5(3)$ & $0.0140(13)$ \\
\hline C10 & $0.5246(9)$ & $0.7809(8)$ & $0.2100(4)$ & $0.0209(15)$ \\
\hline $\mathrm{H} 10$ & 0.423468 & 0.859130 & 0.227785 & $0.025^{\star}$ \\
\hline C11 & $0.6071(10)$ & $0.7732(9)$ & $0.1295(4)$ & $0.0269(17)$ \\
\hline $\mathrm{H} 11$ & 0.572238 & 0.843374 & 0.080144 & $0.032^{\star}$ \\
\hline $\mathrm{C} 12$ & $0.7417(9)$ & $0.6433(9)$ & $0.1290(4)$ & $0.0252(16)$ \\
\hline $\mathrm{H} 12$ & 0.820200 & 0.608317 & 0.079893 & $0.030^{*}$ \\
\hline C13 & $0.7460(8)$ & $0.5723(9)$ & $0.2119(4)$ & $0.0193(15)$ \\
\hline $\mathrm{H} 13$ & 0.828776 & 0.479967 & 0.231279 & $0.023^{*}$ \\
\hline C14 & $0.3558(10)$ & $0.3350(10)$ & $0.2281(4)$ & $0.0276(16)$ \\
\hline $\mathrm{H} 14$ & 0.355028 & 0.295615 & 0.287566 & $0.033^{\star}$ \\
\hline C15 & $0.2391(9)$ & $0.4590(10)$ & $0.1942(5)$ & $0.0296(17)$ \\
\hline $\mathrm{H} 15$ & 0.142143 & 0.523522 & 0.225778 & $0.036^{\star}$ \\
\hline C16 & $0.2846(10)$ & $0.4779(10)$ & $0.1077(5)$ & $0.0309(17)$ \\
\hline $\mathrm{H} 16$ & 0.225493 & 0.556791 & 0.067697 & $0.037^{\star}$ \\
\hline C17 & $0.4283(10)$ & $0.3628(9)$ & $0.0887(4)$ & $0.0265(17)$ \\
\hline H17 & 0.489727 & 0.347099 & 0.032656 & $0.032^{\star}$ \\
\hline C18 & $0.4715(10)$ & $0.2757(9)$ & $0.1627(4)$ & $0.0224(15)$ \\
\hline $\mathrm{H} 18$ & 0.568547 & 0.187502 & 0.167856 & $0.027^{\star}$ \\
\hline C19 & $0.9713(14)$ & $0.1213(13)$ & $0.0828(6)$ & $0.053(2)$ \\
\hline $\mathrm{C} 20$ & $1.0770(13)$ & $0.0124(12)$ & $0.1332(5)$ & $0.056(3)$ \\
\hline $\mathrm{H} 20 \mathrm{~A}$ & 1.002826 & -0.083003 & 0.158746 & $0.084^{\star}$ \\
\hline $\mathrm{H} 20 \mathrm{~B}$ & 1.122269 & 0.079868 & 0.176395 & $0.084^{\star}$ \\
\hline $\mathrm{H} 20 \mathrm{C}$ & 1.178370 & -0.034290 & 0.099169 & $0.084^{\star}$ \\
\hline
\end{tabular}

two symmetry-related $\mathrm{FcCOO}^{-}$, which are asymmetrically bonded in the equatorial plane. This coordination is very similar to the complexes bis $\left(\mathrm{CH}_{3} \mathrm{COO}-\kappa^{2} \mathrm{O}, \mathrm{O}^{\prime}\right)$ bis(DMAP) copper(II) [10], $\operatorname{bis}\left(\mathrm{CH}_{3} \mathrm{COO}-\kappa^{2} \mathrm{O}, \mathrm{O}^{\prime}\right)$ bis(DMAP)copper(II) [11], and bis[ethyl(trifluoroacetyl)acetato- $\kappa^{2} O, O^{\prime}$ ]bis(DMAP)] copper(II) [12].

Acknowledgements: We thank the National Natural Science Foundation of China (21765008), Natural Science Foundation of Guangxi (2017GXNSFBA198178, 2016GXNSFBA380002), the Middle-aged and Young Teachers Basic Ability Promotion Project of Guangxi (2017KY0649).

\section{References}

1. Sheldrick, G. M.: A short history of SHELX. Acta Crystallogr. A64 (2008) 112-122.

2. Brandenburg, K.: DIAMOND. Visual Crystal Structure Information System. Version 3.2i. Crystal Impact, Bonn, Germany (2012).

3. Bruker. APEX3 and SAINT. Bruker AXS Inc., Madison, WI, USA (2012).

4. Nieto, D.; Bruña, S.; González-Vadillo, A. M.; Perles, J.; Carrillo-Hermosilla, F.; Antiñolo, A.; Padrón, J. M.; Plata, G. B.; Cuadrado, I.: Catalytically generated ferrocene-containing guanidines as efficient precursors for new redox-active heterometallic platinum(II) complexes with anticancer activity. Organometallics 34 (2015) 5407-5417.

5. González-Pantoja, J. F.; Stern, M.; Jarzecki, A. A.; Royo, E.; Robles-Escajeda, E.; Varela-Ramírez, A.; Aguilera, R. J.; Contel, M.: Titanocene-phosphine derivatives as precursors to cytotoxic heterometallic $\mathrm{TiAu}_{2}$ and TiM $(\mathrm{M}=\mathrm{Pd}, \mathrm{Pt})$ compounds. studies of their interactions with DNA. Inorg. Chem. 50 (2011) 11099-11110.

6. Patra, M.; Gasser, G.: The medicinal chemistry of ferrocene and its derivatives. Nat. Rev. Chem. 1 (2017) 1-12.

7. Ornelas, C.: Application of ferrocene and its derivatives in cancer research. New J. Chem. 35 (2011) 1973-1985.

8. Liu, K. G.; Shan, Q. D.; Nie, J.; Yan, X. W.: A 2D supramolecular network based on aromatic $\pi \cdots \pi$ stacking interactions. $Z$. Anorg. Allg. Chemie 644 (2018) 82-85.

9. Liu, K. G.; Wang, R.; Han, H. Y.; Nie, J.; Yan, X. W.: Incorporating ferrocenecarboxylato(-1) into a $\left[\mathrm{Cu}_{2}{ }_{2}(\mu-\mathrm{dppm})_{2}\right]^{2+}(\mathrm{dppm}=$ bis (diphenylphosphino)methane) scaffold for hydrogen peroxide sensing. J. Coord. Chem. 71 (2018) 3500-3509.

10. Benslimane, M.; Merazig, H.; Daran, J.: Bis(acetato$\mathrm{K}^{2} \mathrm{O}, \mathrm{O}^{\prime}$ ) bis[4-(dimethylamino)pyridine- $\mathrm{K} N$ ]copper(II). Acta Crystallogr. E67 (2011) m235.

11. Fomina, I.; Dobrokhotova, Z.; Aleksandrov, G.; Bogomyakov, A.; Fedin, M.; Dolganov, A.; Magdesieva, T.; Novotortsev, V.; Eremenko, I.: Influence of the nature of organic components in dinuclear copper(II) pivalates on the composition of thermal decomposition products. Polyhedron 29 (2010) 1734-1746.

12. Patev, N.; Císařová, I.; Zdravkova, M.; Vohlídal, J.: Structure of bis[ethyl(trifluoroacetyl)acetato]copper(II) and its adduct with 4-(dimethylamino)pyridine: EPR and X-ray study. J. Mol. Struct. 689 (2004) 191-197. 